\title{
Localização-alocação de centros de integração logística submetidos a demandas par-a-par
}

\author{
Vanessa de Almeida Guimarães ${ }^{1}$, Glaydston Mattos Ribeiro², Vinicius Leal do Forte ${ }^{3}$, Abilio Lucena ${ }^{4}$, \\ Artur Monteiro Leitão Junior ${ }^{5}$, Luiz Carlos de Souza Neves Pereira ${ }^{6}$ e Francielle Avancini Fornaciari ${ }^{7}$
}

\begin{abstract}
Resumo: A partir do lançamento do Plano Nacional de Logística e Transporte - PNLT, o planejamento estratégico do setor de transportes brasileiro passou a apontar para a necessidade de instalação de Centros de Integração Logística - CILs; visando promover a intermodalidade e, consequentemente, aumentar tanto a eficiência do setor quanto a competitividade do país. Este trabalho investiga o uso de um modelo matemático de localização-alocação para o problema. O modelo se baseia no atendimento explícito de demandas par-a-par e na minimização dos custos logísticos envolvidos. Foi testado considerando um cenário que envolve quatro produtos economicamente relevantes e utilizando a base de dados georreferenciada do PNLT, estimada para o ano de 2023. Os resultados obtidos propõem a abertura de $10 \mathrm{CILs}$, reduzindo custos de transporte e gerando ganhos de eficiência para o setor, como um todo.
\end{abstract}

Palavras-chave: otimização, centro de integração logística, planejamento de transporte.

Abstract: After the publication of the Brazilian National Logistics and Transportation Plan (PNLT), the strategic planning for the Brazilian transportation sector has indicated the need for Logistics Integration Centers - CILs so as to promote intermodal transportation and, as a result, obtain efficiency gains and competitiveness for the country. This paper investigates the use of a location-allocation mathematical model for the problem that takes into account peer-to-peer demands and minimizes the total logistics costs. The model was tested on a problem instance that involves four economically relevant products and that relies on data originating from the PNLT, estimated for the year 2023. As a result, the model suggests the opening of 10 CILs, thus reducing transportation costs and enhancing the efficiency of the transportation sector.

Keywords: optimization, logistic integration center, transport planning.

\section{INTRODUÇÃO}

A dimensão territorial do Brasil aliada à dispersão geográfica de suas atividades produtivas faz com que o setor de transportes tenha fundamental importância para o desenvolvimento econômico das mais diversas regiões produtoras e consumidoras do país (CNT e COPPEAD, 2002; Fleury, 2003; Diniz, 2006). Enfatizando esse ponto, Padula (2008) destaca que a infraestrutura de transportes é essencial para integração entre os mercados produtores e consumidores, internos e/ou externos, e que um aumento de eficiência neste setor se propaga por toda a cadeia produtiva.

No caso específico do Brasil, a baixa eficiência do setor de transportes pode ser constatada, dentre outros fatores, pelo fato do custo logístico representar de 10,6\% a $15,4 \%$ do valor do PIB nacional, comparado aos $8,5 \%$ relativos aos Estados Unidos (CNT, 2014). Disto resulta, em parte, o chamado "Custo Brasil", calculado com base nas restrições legais, institucionais, burocráticas e de infraestrutura que condicionam as atividades econômicas e sociais e

\footnotetext{
1 Vanessa de Almeida Guimarães, Centro Federal de Educação Tecnológica Celso Suckow da Fonseca - CEFET/RJ e Programa de Engenharia de Transportes - PET/COPPE/UFRJ.

(vanessaguim@hotmail.com)

2 Glaydston Mattos Ribeiro, Programa de Engenharia Transportes,

PET/COPPE/UFRJ. (glaydston@pet.coppe.ufrj.br)

3 Vinicius Leal do Forte, Departamento de Matemática, UFRRJ.

(vlforte@gmail.com)

${ }^{4}$ Abilio Lucena, Programa de Engenharia de Sistemas e Computação,

PESC/COPPE/UFRJ. (abiliolucena@cos.ufr.br)

${ }^{5}$ Artur Monteiro Leitão Junior, Ministério dos Transporte, SPNT/MT.

(artur.junior@transportes.gov.br)

${ }^{6}$ Luiz Carlos de Souza Neves Pereira, Ministério dos Transporte,

SPNT/MT. (luiz.pereira@transportes.gov.br)

${ }^{7}$ Francielle Avancini Fornaciari, Ministério dos Transporte, SPNT/MT.

(francielle.fornaciari@transportes.gov.br)
}

Manuscrito recebido em 24/10/2015 e aprovado para publicação em 03/08/2016.

Este artigo é parte de TRANSPORTES v. 25, n. 1, 2017. ISSN: 2237-1346 (online). DOI:10.14295/transportes.v25i1.1027 que limitam o crescimento econômico (CNT, 2014).

Motivado pelo exposto acima, o planejamento estratégico do setor de transporte de cargas brasileiro tem focado na redução de custos, o que levaria a um aumento de competitividade para o país. Nesse sentido, programas do governo federal, como o Plano Nacional de Logística e Transporte - PNLT (MT, 2007, 2009, 2012), apontam para o uso da intermodalidade e sugerem ações para equilibrar a matriz de transporte de cargas do país. Tais ações, por sua vez, levariam à almejada redução de custos, com a utilização de modos de transporte de maior capacidade. Entretanto, para se alcançar a intermodalidade é necessário implantar, dentre outros, estruturas de integração logística, como os Centros de Integração Logística (CILs) que aqui serão investigados.

Este estudo utilizou-se do modelo matemático de localização e dimensionamento de CILs proposto por Guimarães (2015), que minimiza custos logísticos considerando explicitamente os fluxos de carga entre origens e destinos, definidos por demandas par-a-par. Para testar a adequação do modelo, experimentos computacionais foram efetuados restringindo a localização de CILs ao território nacional e considerando apenas o transporte de quatro produtos agrícolas. A saber, soja, açúcar, farelo e milho, que figuram entre os principais produtos do agronegócio brasileiro (MA, 2015) e envolvem volumes de carga expressivos (MT, 2007). Como uma delimitação adicional ao estudo, ressaltase que foi usada a base de dados georreferenciada do PNLT, estimada para o ano de 2023 (MT, 2007), e também as projeções da infraestrutura de transportes por ele sugeridas.

Assim, em relação aos procedimentos metodológicos, esta pesquisa se caracteriza por ser de natureza aplicada que, de acordo com Silva e Menezes (2001), tem como objetivo gerar conhecimento para solucionar de forma prática um problema específico. Para tanto, foram feitas pesquisas bibliográfica e documental, as quais permitiram compreender a configuração do setor de transporte brasileiro e os modelos matemáticos que poderiam ser aplicados para solução 
do problema objeto de estudo. Em seguida, selecionou-se o modelo considerado mais aderente ao escopo do trabalho e aplicou-o à base de dados para geração dos resultados. $\mathrm{O}$ tratamento de dados foi feito de maneira quantitativa (aplicação da modelagem matemática) e qualitativa (descrição e discussão dos resultados).

Além desta introdução, o artigo contém cinco seções adicionais. Na Seção 2 é descrita a relação entre planejamento de transportes e estruturas de integração logística. Em seguida, na Seção 3, é apresentado o modelo matemático utilizado. A definição dos parâmetros do modelo aplicados ao estudo de caso proposto é feita na Seção 4. Por sua vez a análise dos dados de entrada e os resultados computacionais obtidos são discutidos na Seção 5. Finalmente, conclusões e sugestões para trabalhos futuros são apresentados na Seção 6.

\section{O PLANEJAMENTO DE TRANSPORTE E SUA RELAÇÃO COM ESTRUTURAS DE INTEGRAÇÃO}

Lopes et al. (2008) analisaram possíveis alternativas para melhorar a eficiência do transporte de cargas no Brasil. Dentre elas, os autores sugerem que o planejamento estratégico do setor deve focar na redução dos custos de movimentação de carga. Corroborando esta tese, o relatório da CNT (2014) destaca que algumas lacunas encontradas no planejamento e desenvolvimento de um sistema de transporte integrado para o país têm gerado desequilíbrios na matriz de transporte brasileira. Isto, por sua vez, tem levado ao aumento dos custos de movimentação de cargas, impactando assim na competitividade do país e aumentando o "Custo Brasil".

Relacionados direta ou indiretamente às observações acima, diferentes planos e programas federais e estaduais foram lançados ao longo das duas últimas décadas, sinalizando a necessidade de investimentos específicos em infraestrutura de transporte (ampliação ou implantação). Dentre estes, destaca-se o PNLT, lançado em 2007 e revisado em 2009 e 2011, que fomenta a inter/multimodalidade e o reequilíbrio da matriz de transporte de cargas ao combinar as eficiências de diferentes modos de transporte (MT, 2007, 2009 e 2012).

Neste ponto, ressalta-se que a diferença entre intermodalidade e multimodalidade reside na responsabilidade do transportador. De acordo com Moori e Riquete (2014) e Agência Nacional de Transporte Terrestre - ANTT (2016): o transporte intermodal requer a emissão de um documento para cada uma das atividades executadas (transporte rodoviário, aquaviário e transbordo, por exemplo); enquanto no multimodal, existe a figura do Operador do Transporte Multimodal - OTM (regulamentado pela Lei n. 9.611/1998 e pelo Decreto $\left.n^{\circ} 3411 / 2000\right)$ que é responsável pelo transporte, da origem até o destino, o qual é regido por um único contrato (Conhecimento do Transporte Multimodal de Carga).

Ressalta-se que o PNLT possui características de Plano Plurianual que estima as matrizes de produção e consumo brasileiras para diferentes tipos de produtos (para os anos de 2015, 2019, 2023, 2027 e 2031) e sugere intervenções de infraestrutura visando alcançar o reequilíbrio da matriz de transportes. Correa e Ramos (2010), Fleury (2011), Costa (2014) e Quadros e Nassi (2015) apontam-no como a retomada do planejamento estratégico no setor de transportes brasileiro, após um longo período de falta de investimentos coordenados e de integração sistêmica.

Após o PNLT, diferentes planos e programas foram lançados pelos governos estaduais e federais (conforme evidenciado em Guimarães, 2015), dentre os quais se destaca o Programa de Investimento em Logística - PIL (EPL, 2014). Lançado em 2012, o PIL tem os seguintes objetivos: (i) elevar a escala dos investimentos em infraestrutura de transporte, dotando o país de uma rede considerada "ampla, moderna e com tarifas módicas"; (ii) promover a intermodalidade e integração; e (iii) permitir a articulação com as cadeias produtivas. Seu horizonte de planejamento se estende ao ano de 2042, visando prover melhorias às infraestruturas rodoviária, portuária, ferroviária e aérea.

Entretanto, para que se possa usufruir da intermodalidade e integração almejadas pelos planos de transporte e logística, além de investimentos em infraestrutura, é necessário destinar recursos para instalação (e/ou adequação) de CILs, ou seja, estruturas de integração logística. Estas podem variar em complexidade, indo de terminais de transbordo simplificados até plataformas logísticas que permitem a agregação de valor aos produtos movimentados (Guimarães, 2015). Contudo, para que conduzam, de fato, a uma redução de custos, os CILs devem estar localizados em pontos estratégicos do território nacional.

Sirikijpanichkul e Ferreira (2005) ressaltam que a adequada localização de terminais é essencial para o sucesso do transporte de cargas intermodal. Da mesma forma, Daskin et al. (2003) consideram-na crítica também para a eficiência da cadeia de suprimentos, uma vez que decisões sobre transporte e formação de estoque são estratégicas para o desempenho empresarial. Acrescente-se a isso o fato de que mudar a localização de um terminal é algo que requer tempo (estratégia de longo prazo) e recursos financeiros, além de gerar inconveniências para os clientes (Slack et al., 2009). Devido à complexidade de bem localizar e dimensionar CILs, modelos matemáticos e algoritmos de solução, em particular aqueles associados à Otimização Matemática, são então fundamentais para cumprir a tarefa.

$\mathrm{Na}$ literatura foram encontrados estudos sobre a localização de $h u b s$ intermodais, ou seja, um dos possíveis tipos de CILs (Arnold et al., 2004; Groothedde et al., 2005; Racunica e Wynter, 2005; Almur e Kara, 2008; Limbourg e Jourquin, 2009; Ishfaq e Sox, 2011; Almur et al., 2012; Campbell e O'Kelly, 2012; Farahani et al., 2013). Em particular, as duas últimas referências consideram redes huband-spoke e indicam, entre outros, Groothedde et al. (2005), Limbourg e Jourquin (2009), Vasconcelos et al. (2011) e Vidovic et al. (2011) para o caso específico de redes intermodais.

Além disso, levantaram-se estudos sobre localização de terminais intermodais, tais como: Crainic e Laporte (1997), Bhattacharya et al. (2014) e Sirikijpanichkul e Ferreira (2005). Ressalta-se que os trabalhos de Crainic e Laporte (1997), Almur e Kara (2008), Melo et al. (2009) e Campbell e O'Kelly (2012) fazem uma extensa revisão de literatura sobre o tema, evidenciando a importância da discussão feita neste artigo.

Considerando apenas a etapa de alocação, pode-se citar o trabalho de Meisel et al. (2013), que considera restri- 
ções de capacidade para os pontos de origem e envolve decisões sobre o transporte intermodal de uma cadeia de suprimentos (terminais de transbordo definidos a priori). Especificamente para localização-alocação, destacam-se três modelos: Dubke (2006), Costa (2014) e Guimarães (2015).

Dubke (2006) propõe um modelo matemático de localização-alocação de terminais logísticos, aplicando-o ao escoamento da soja brasileira. A autora considera agregação de valor à soja. Ou seja, trabalha com um produto de entrada no CIL - soja a granel - e três produtos de saída - soja, farelo e óleo de soja. Entretanto, não são consideradas as demandas par-a-par. Entende-se por demanda par-a-par que os pontos de destino (consumidores) serão atendidos pelos pontos de origem (produtores) com os quais mantém relação comercial ou que sejam de sua preferência, não cabendo a um terceiro (governo, gestor do CIL etc.) intervir nesta relação de mercado.

O modelo de Costa (2014) define locais para a implantação de CILs, dadas as configurações da rede de transporte conectando pontos de produção a pontos de consumo. Entretanto, ele permite que demandas distintas de um mesmo produto sejam eventualmente combinadas dentro de um CIL, não respeitando assim o atendimento explícito das demandas par-a-par. O trabalho de Guimarães (2015), por sua vez, refina o trabalho de Costa (2014) ao impor o atendimento explícito das demandas par-a-par.

Dentre os modelos levantados, este é o primeiro a contemplar o atendimento explícito de demandas par-a-par. Embora negligenciado nos demais trabalhos, tal funcionalidade é importante, pois permite ao comprador receber exatamente o que encomendou e não uma alternativa do mesmo produto, enviada de outra origem e com uma especificação eventualmente distinta da desejada.

Posto isto, o modelo de Guimarães (2015) foi selecionado para a investigação aqui conduzida, que é descrita em detalhes na Seção 3. Finalmente, ao concluir esta seção, indica-se o texto de Melo et al. (2009) para uma resenha sobre a área de investigação aqui considerada.

\section{MODELAGEM MATEMÁTICA DO PROBLEMA DE LOCALIZAÇÃO-ALOCAÇÃO DE CILS}

Com base no modelo desenvolvido por Costa (2014), Guimarães (2015) propôs um modelo que respeita as demandas par-a-par. Estas, por sua vez, aparecem identificadas nas matrizes de origem-destino de carga do PNLT. Ou seja, se um determinado produto deve deixar um local de produção e chegar a um local de consumo específico, existe então uma relação biunívoca entre o que é enviado e o que é recebido, não sendo permitidas alterações de qualquer espécie ao longo do trajeto. Isto se aplica mesmo que o deslocamento envolva a utilização de uma estrutura concentradora de carga, do tipo CIL.

Tal característica do modelo de Guimarães (2015) o torna aderente a uma realidade econômica em que: (1) fornecedores de bens e serviços preocupam-se em atender clientes específicos e/ou a respeitar contratos já estabelecidos; e (2) pode haver relação de confiança entre produtores e consumidores, não estando os clientes dispostos a ter sua demanda satisfeita por outros fornecedores. Destaca-se ainda que, para efeito de modelo, os fluxos de produtos podem ser considerados de duas maneiras: fluxo direto entre um par de origem e destino (O/D) e o fluxo tendo um CIL como um ponto intermediário entre a origem e o destino.

Para descrever o modelo, seja I um conjunto de pontos de produção, $J$ um conjunto de pontos de consumo, $P$ um conjunto de produtos e $K$ um conjunto de locais candidatos à abertura de CILs. Têm-se, então, os seguintes parâmetros:

- $M_{p i}^{\text {dir }}$ : conjunto dos modos disponíveis para transportar diretamente o produto $p$ do ponto de produção $i$ para o ponto consumidor $j, p \in P, i \in I$ e $j \in J$;

- $M_{p i k}^{\text {ent }}$ : conjunto de modos disponíveis para transportar o produto $p$ do ponto de produção $i$ para o candidato a CIL $k, p \in P, i \in I$ e $k \in K$;

- $M_{p k j}^{s a i}$ : conjunto de modos disponíveis para transportar o produto $p$ do candidato a CIL $k$ para o ponto de consumo $j, p \in P, k \in K$ e $j \in J$;

- $C_{p i j m}^{d i r}$ : custo unitário de transporte do produto $p$ da zona de produção $i$ para o ponto de consumo $j$, de maneira direta pelo modo de transporte $m$, ou seja, sem a utilização do CIL, $p \in P, i \in I, \quad j \in J$ e $m \in M$;

- $C_{p i k m}^{e n t}$ : custo unitário de transporte do produto $p$ da zona de produção $i$ para o candidato a CIL $k$, pelo modo de transporte $m, \quad p \in P, \quad i \in I, \quad k \in K$ e $m \in M_{p i k}^{\text {ent }}$;

- $C_{p k j m}^{s a i}$ : custo unitário de transporte do produto $p$ do candidato a CIL $k$ até a zona de consumo $j$, pelo modo de transporte $m, \quad p \in P, k \in K, j \in J$ e $m \in M_{p k j}^{s a i}$;

- $C T_{p k j m}^{s a i}$ : custo do(s) transbordo(s) do produto $p$, entre o candidato a CIL $k$ e a zona de consumo $j$ para o modo $m, p \in P, k \in K, j \in J$ e $m \in M_{p k j}^{s a i}$. Este custo está relacionado à troca modal proporcionada pelo CIL, tendo em vista que mais de uma troca modal pode ser feita ao longo do trajeto entre $k$ e $j$, desde que proporcione redução no custo de transporte;

- $g_{p k}$ : custo fixo de utilização do candidato a CIL $k$ pelo produto $p, k \in K$ e $p \in P$;

- $\alpha_{p k}$ : custo variável de utilização do candidato a CIL $k$ pelo produto $p, k \in K$ e $p \in P$;

- $v_{k}^{\min }$ : representa o volume mínimo para abertura do candidato a CIL $k \in K$;

- $v_{k}^{\max }:$ representa a capacidade máxima do candidato a CIL $k \in K$; e

- $d_{i p j}$ : refere-se à quantidade total do produto $p$ demandada pela zona de consumo $j$, proveniente da zona de produção $i, p \in P, j \in J$ e $i \in I$.

Por sua vez, as variáveis de decisão utilizadas no modelo são representadas por: 
- $x_{p i j m}^{d i r}$ : volume do produto $p$ transportado diretamente do ponto de produção $i$ até o ponto de consumo $j$ pelo modo $m, p \in P, \quad i \in I, j \in J$ e $m \in M_{p i j}^{d i r}$

- $x_{\text {pikm }}^{\text {ent }}$ : volume do produto $p$ transportado do ponto de produção $i$ até o candidato a CIL $k$ pelo modo $m$ (representa fluxo de entrada de produtos no CIL), $p \in P, i \in I, k \in K$ e $m \in M_{p i k}^{\text {ent }}$;

- $x_{p k j m}^{s a i}$ : volume do produto $p$ transportado do candidato a CIL $k$ até o ponto de consumo $j$ pelo modo $m$ (representa o fluxo de saída do CIL), $p \in P$, $j \in J, k \in K$, e $m \in M_{p k j}^{s a i}$;

- $z_{k}$ : variável binária que indica se o candidato a CIL $k \in K$ deve ser aberto $\left(z_{k}=1\right)$ ou não $\left(z_{k}=0\right)$;

- $y_{p i k j}$ : refere-se ao volume de produto $p$ transportado de $i$ para $j$, que utiliza o candidato a CIL $k, p \in P$, $i \in I, j \in J$ e $k \in K$. Trata-se de uma variável de decisão que garante que as demandas par-a-par sejam respeitadas, ou seja, que determinado produto $p$ demandado por uma zona $i$ específica, será atendida por esta zona $j$, mesmo quando enviada por meio de um CIL.

O modelo de Programação Linear Inteira Mista, proposto para o problema, tem então a seguinte formulação:

\section{Minimizar $v(C I L)=$}

$$
\begin{aligned}
& \sum_{p \in P} \sum_{i \in I} \sum_{j \in J} \sum_{m \in M_{p i j}^{d i r}} C_{p i j m}^{d i r} x_{p i j m}^{d i r}+\sum_{p \in P} \sum_{i \in I} \sum_{k \in K} \sum_{m \in M_{p i k}^{e n t}} C_{p i k m}^{e n t} x_{p i k m}^{e n t}+ \\
& \sum_{p \in P} \sum_{k \in K}\left(g_{p k} z_{k}+\alpha{ }_{p k} \sum_{j \in J} \sum_{m \in M_{p k j}^{s a i}} x_{p k j m}^{s a i}\right)+ \\
& \sum_{p \in P} \sum_{k \in K} \sum_{j \in J} \sum_{m \in M_{p k j}^{s a i}}\left(C_{p k j m}^{s a i}+C T_{p k j m}^{s a i}\right) x_{p k j m}^{s a i}
\end{aligned}
$$

\section{Sujeito a:}

$$
\begin{aligned}
& \sum_{m \in M_{p i j}^{d i r}} x_{p i j m}^{d i r}+\sum_{k \in K} y_{p i k j}=d_{i p j} \forall p \in P, i \in I, j \in J \\
& v_{k}^{\min } z_{k} \leq \sum_{p \in P} \sum_{i \in I} \sum_{m \in M_{p i k}^{e n}} x_{p i k m}^{e n t} \leq v_{k}^{\max } z_{k} \forall k \in K \\
& \sum_{i \in I} y_{p i k j}=\sum_{m \in M_{p h j}^{e n t}} x_{p i k m}^{e n t} \forall i \in I, p \in P, k \in K \\
& \sum_{i \in I} y_{p i k j}=\sum_{m \in M_{p p j}^{s a j}} x_{p k j m}^{s a i} \quad \forall j \in J, p \in P, k \in K \\
& x_{p i j m}^{d i r} \geq 0 \quad \forall p \in P, i \in I, j \in J, m \in M_{p i j}^{d i r} \\
& x_{p i k m}^{e n t} \geq 0 \quad \forall p \in P, i \in I, k \in K, m \in M_{p i k}^{e n t}
\end{aligned}
$$

$$
\begin{aligned}
& x_{p k j m}^{s a i} \geq 0 \forall p \in P, k \in K, j \in J, m \in M_{p k j}^{s a i} \\
& y_{p i k j} \geq 0 \forall p \in P, i \in I, k \in K, j \in J \\
& Z_{k} \in\{0,1\} \forall k \in K
\end{aligned}
$$

A Função Objetivo (1) minimiza os custos de transporte, considerando para tanto os custos de transporte direto e aqueles envolvendo o uso de CILs. Leva em conta, também, os custos operacionais (fixos e variáveis) destas estruturas e seus custos de transbordo. As Restrições (2) garantem que a demanda total de cada zona de consumo será atendida, seja por transporte direto entre a zona de produção e a de consumo, seja passando por um CIL. Garante também que, independente do CIL eventualmente utilizado, as demandas par-a-par serão respeitadas. As Restrições (3) estão relacionadas às capacidades máximas e mínimas de utilização de CILs e impactam diretamente na decisão de abri-los ou não.

As Restrições (4) impõem que o volume total de um produto que deixa uma zona de produção e se destina a uma zona de consumo passando por um CIL será exatamente igual ao volume total do produto que entra no CIL tendo como origem a zona de produção. Isso significa dizer que todo o volume de um dado produto que entra no CIL já tem, de antemão, uma destinação específica, não podendo ser usado para atender a demanda de outra zona de consumo. As Restrições (5) complementam as Restrições (4) e estão associadas aos fluxos de saída. As demais restrições estão relacionadas ao domínio de definição das variáveis.

Vale ressaltar que o problema aqui descrito é eminentemente estratégico, agregando, entretanto, características de um problema tático. Salienta-se que o modelo proposto por Guimarães (2015) generaliza o problema de localização de facilidades capacitado multiproduto multicamada (PLFCMM), em particular, o modelo proposto por Geoffrion e Graves (1974). Isso é feito, dentre outros aspectos, ao estendê-lo para o atendimento de demandas par-a-par.

Em termos de complexidade computacional, o PLFCMM é NP-difícil (para detalhes, veja Geoffrion e Graves, 1974) e, assim sendo, o problema de localização-alocação de CILs também o é. Isso significa dizer que inexistem algoritmos de solução eficientes para o problema em análise. Significa também que, embora tenham sido resolvidos muitos casos práticos do modelo à otimalidade, em tempos de processamento computacional aceitáveis, nenhuma garantia existe de que isso sempre venha a acontecer.

Uma nota final é que, vencidos os desafios descritos acima, elementos de Programação Estocástica (Shapiro, Dentcheva e Ruszczyński, 2009) que contemplam incertezas em relação aos dados de entrada do modelo, deveriam ser também explorados. A sugestão se aplica já que aqui trabalhamos com projeções de demandas futuras. No entanto, ao fazê-lo, a alta complexidade computacional associada a modelos de Programação Estocástica deve ser também levada em consideração. 


\section{ESPECIFICAÇÃO DOS PARÂMETROS E DOS DADOS DE ENTRADA DO MODELO ADOTADOS PARA O ESTUDO DE CASO}

Para testar o modelo da Seção 3, foram utilizados os seguintes dados de entrada:

1.O território brasileiro foi dividido em 559 zonas de produção e consumo (microrregiões) e acrescido de uma zona no exterior, de acordo com os critérios definidos pelo PNLT (MT, 2007);

2. Foi utilizada a rede de transportes e a base de dados georreferenciada do PNLT para o ano de 2023. Para tanto, foram identificadas as conexões intermodais (rodoviário, hidroviária e/ou ferroviária) e os terminais de transbordo (que possibilitam a intermodalidade);

3. Foram selecionados quatro produtos para o experimento (vide Seção 1): soja, farelo de soja, milho e açúcar. Tais produtos pertencem ao Grupo 1 (categorizado na revisão do PNLT de 2012), sendo estes os de maior potencial de intermodalidade (MT, 2012; Guimarães, 2015), no grupo. Feita a escolha, suas matrizes de produção e consumo estimadas para o ano de 2023 pelo PNLT (MT, 2007) foram utilizadas;

4. Como custos associados aos CILs, consideraram-se um custo fixo relacionado à instalação da estrutura e um custo variável, por volume processado. Estes foram estimados por meio de dados coletados das seguintes fontes: BNDES (2011), ANTT (2012 apud Costa 2014), USP (2012), ANTAQ (2013), CONAB (2013 apud Costa 2014), e Costa (2014). Destaca-se que, para o custo fixo, considerou-se o valor simbólico adotado por Costa (2014), para identificar aquelas microrregiões que estrategicamente comportariam um CIL;

5.Para efeito da aplicação, os CILs foram considerados terminais de transbordo simplificados, ou seja, permitem apenas a consolidação e a transferência modal. Não trazem, portanto, agregação de valor ou qualquer serviço adicional;

6. Considerou-se 1.000.000 toneladas como volume mínimo exigido para abertura de um CIL, conforme Costa (2014). O volume máximo permitido foi deixado livre, já que depende da capacidade ociosa de ferrovias e hidrovias;

7. Não foram considerados custos fixos de instalação, tendo em vista enfatizar um viés de planejamento estratégico de transportes sob uma ótica governamental, pressupondo, então, que o governo instalaria a estrutura independente de seu custo; e

8. Foram pré-selecionadas regiões potenciais para instalação de CILs. Para tanto, considerou-se: (i) a existência de entroncamentos modais que possibilitassem a intermodalidade; e (ii) a identificação das regiões previstas para concentrar, em 2023, 80\% da oferta dos quatro produtos selecionados (MT, 2007; Costa, 2014). Ao final, 80 microrregiões foram escolhidas como candidatas à instalação de CILs. Vale notar que proximidade das zonas de produção (potenciais clientes do CIL) foi considerada, ainda que indiretamente, neste processo de seleção. Isto, por sua vez, está em consonância com as sugestões propostas por Chopra e Meindl (2011).
Destaca-se que, para efeito de simplificação, considerou-se que o transporte de determinado produto de uma zona de produção para uma zona de consumo tem, em geral, dois tipos de caminhos possíveis: (i) a transferência direta entre a origem e o destino, feita exclusivamente pelo modo rodoviário; e (ii) transporte por meio de um CIL, chegando a ele exclusivamente pelo modo rodoviário e de lá saindo por um modo distinto.

\section{APRESENTAÇÃO E DISCUSSÃO DOS DADOS}

Um código na linguagem $\mathrm{C}++$ foi escrito para gerar uma entrada de dados para o software de Programação Inteira Mista IBM ILOG CPLEX 12.6, relativos ao modelo matemático da Seção 3 e ao estudo de caso descrito na Seção 4. Deste processo resultou uma quantidade excessiva de variáveis e de restrições, impossibilitando a resolução direta do modelo pelo software, em um computador com os seguintes atributos: processador Intel Xeon $3.16 \mathrm{Ghz}, 8 \mathrm{~GB}$ de memória RAM e sistema Operacional Ubuntu 12.04.5 LTS. Procedeu-se, então, a um pré-processamento do modelo visando eliminar variáveis e restrições redundantes ou irrelevantes, conforme descrito a seguir:

a. Foram eliminadas todas as variáveis $x_{p i j m}^{d i r}, x_{p i k m}^{e n t}$, $x_{p k j m}^{s a i}$ e $y_{p i k j}$ que não estivessem associadas a uma demanda par-a-par; e

b. Se o custo de transporte direto de um produto entre a zona de produção e a de consumo é menor que o custo de transporte via CIL, descarta-se então a segunda opção. Elucida-se que, neste artigo, um CIL é uma estrutura de integração que promove o transporte intermodal. Sendo assim, promover a troca modal para alternativas de maior capacidade (como ferrovias ou hidrovias), pode-se obter um custo total menor do que o transporte unimodal (especialmente se este for rodoviário), em função do ganho de escala para o transporte de quantidades significativas.

Finalmente, vale notar que um produto não será necessariamente transportado pelo candidato a CIL que lhe é mais favorável. Este, eventualmente, pode não vir a ser aberto. Os resultados obtidos no pré-processamento são descritos no Quadro 1.

Quadro 1. Comparação do número de variáveis do modelo sem e

\begin{tabular}{|c|c|c|}
\multicolumn{3}{|c|}{ com pré-processamento } \\
\hline Variáveis & Original & Com pré-processamento \\
\hline$x_{p i j m}^{\text {dir }}$ & 1.249 .924 & 46.362 \\
\hline$x_{p i k m}^{\text {ent }}$ & 178.880 & 7.265 \\
\hline$x_{p k j m}^{\text {sai }}$ & 178.880 & 7.912 \\
\hline$y_{i p k j}$ & 99.993 .920 & 28.605 \\
\hline$z$ & 80 & 80 \\
\hline $\begin{array}{c}\text { Tempo de } \\
\text { solução com o } \\
\text { CPLEX }\end{array}$ & Não resolvido & 0,4 segundos \\
\hline
\end{tabular}




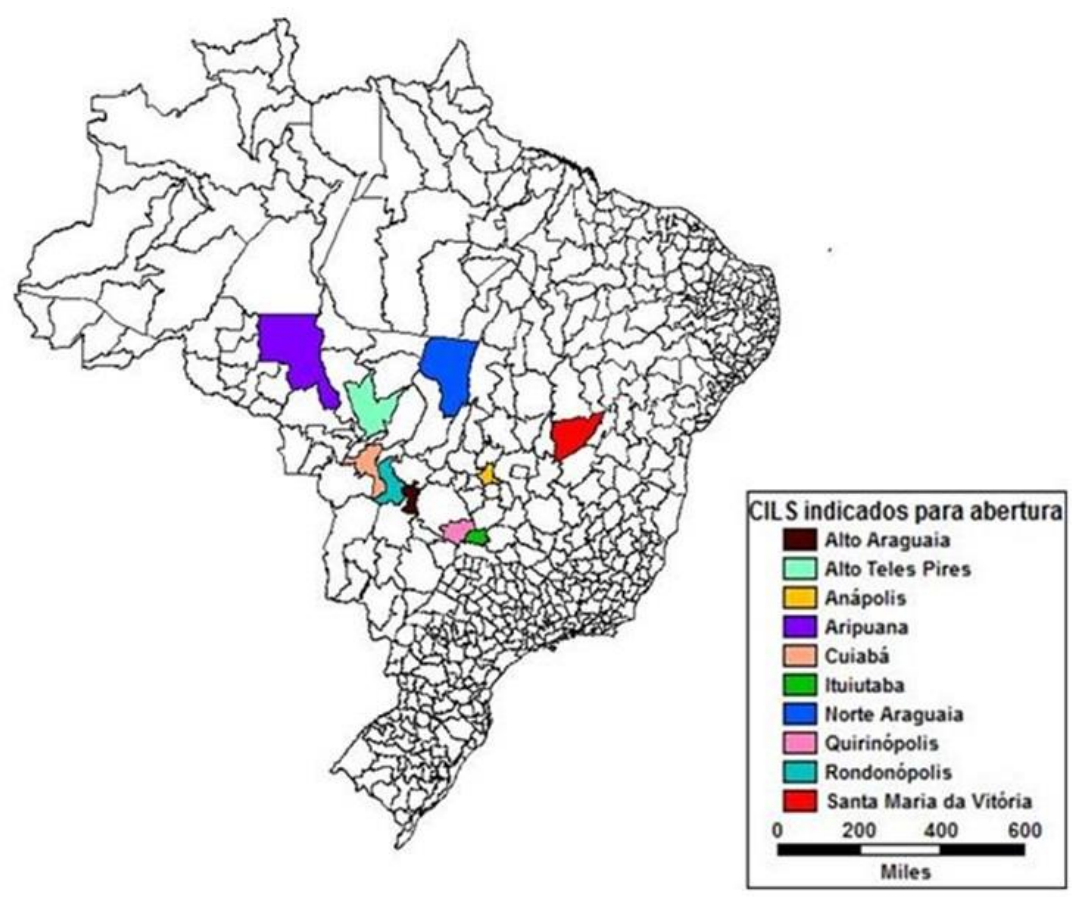

Figura 1. Localização geográfica dos CILs indicados para abertura

O modelo (1)-(10), sob os parâmetros e dados de entrada sugeridos na Seção 4, propõe a abertura de 10 (dez) CILs. Suas localizações geográficas estão indicadas na Figura 1. Nota-se que, com exceção das microrregiões de Santa Maria de Vitória - BA e Ituiutaba - MG, todas as demais localidades sugeridas se concentram na região centro-oeste, o que pode ser explicado pela proximidade com as zonas de produção (conforme MT, 2007).

Ao todo, são movimentadas mais de 42 milhões de toneladas (t), representando para a soja $45 \%$ do volume total transportado por CILs e $16 \%$ do total movimentado em 2023 , seguida pelo milho (23\% passando por CILs e $13 \%$ do total), pelo farelo ( $22 \%$ e $17 \%$, respectivamente) e pelo açúcar (10\% e 8\%, respectivamente). A distribuição dos volumes totais movimentados por CIL é descrita na Figura 2.

Verifica-se que o CIL com o maior movimentação de carga é o da microrregião de Aripuanã, representando $45,1 \%$ do volume total transportado por meio de CILs. A seguir temos: Quirinópolis e Rondonópolis, com 12,2\% e $11 \%$, respectivamente. As demais estruturas têm um volume de movimentação abaixo de 4 milhões de t. Destacase que os CILs das microrregiões de Ituiutaba, Araguaia e Anápolis movimentam exatamente a quantidade mínima exigida para implantá-los, processando, cada um deles, $2,4 \%$ do volume total transportado por CILs.

Divididos produto a produto, os volumes processados por cada CIL são descritos em termos percentuais na Figura 3. A soja é o produto com maior movimentação nos CILs das microrregiões definidas por Santa Maria da Vitória $(60,3 \%)$, Ituiutaba $(92,5 \%)$, Aripuanã $(68,5 \%)$ e Quirinópolis $(37,8 \%)$. O milho, por sua vez, se destaca em Alto Teles Pires $(63 \%)$ e Anápolis $(38,5 \%)$ enquanto o farelo é o produto mais representativo em Cuiabá (85,8\%), Rondonópolis $(81,5 \%)$ e Alto Araguaia $(69,2 \%)$. Já o açúcar é o produto que se destaca no CIL da microrregião de Norte Araguaia $(45 \%)$

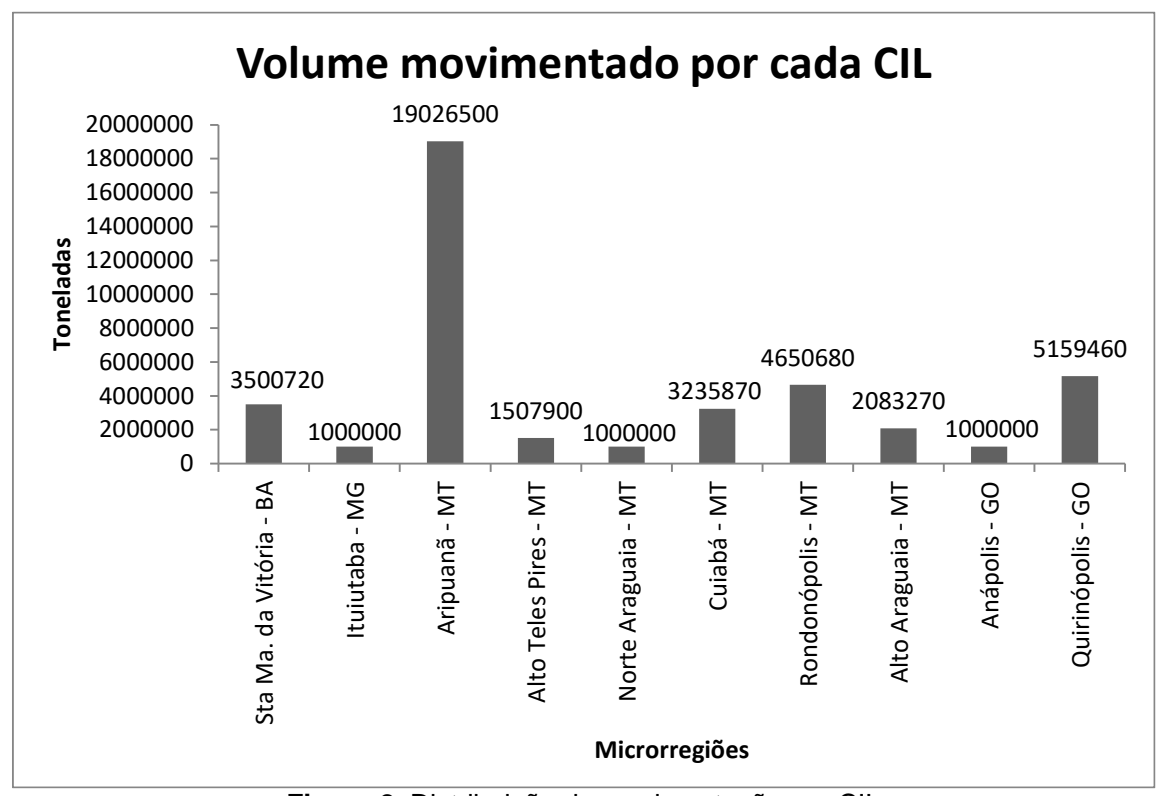

Figura 2. Distribuição da movimentação por CIL 


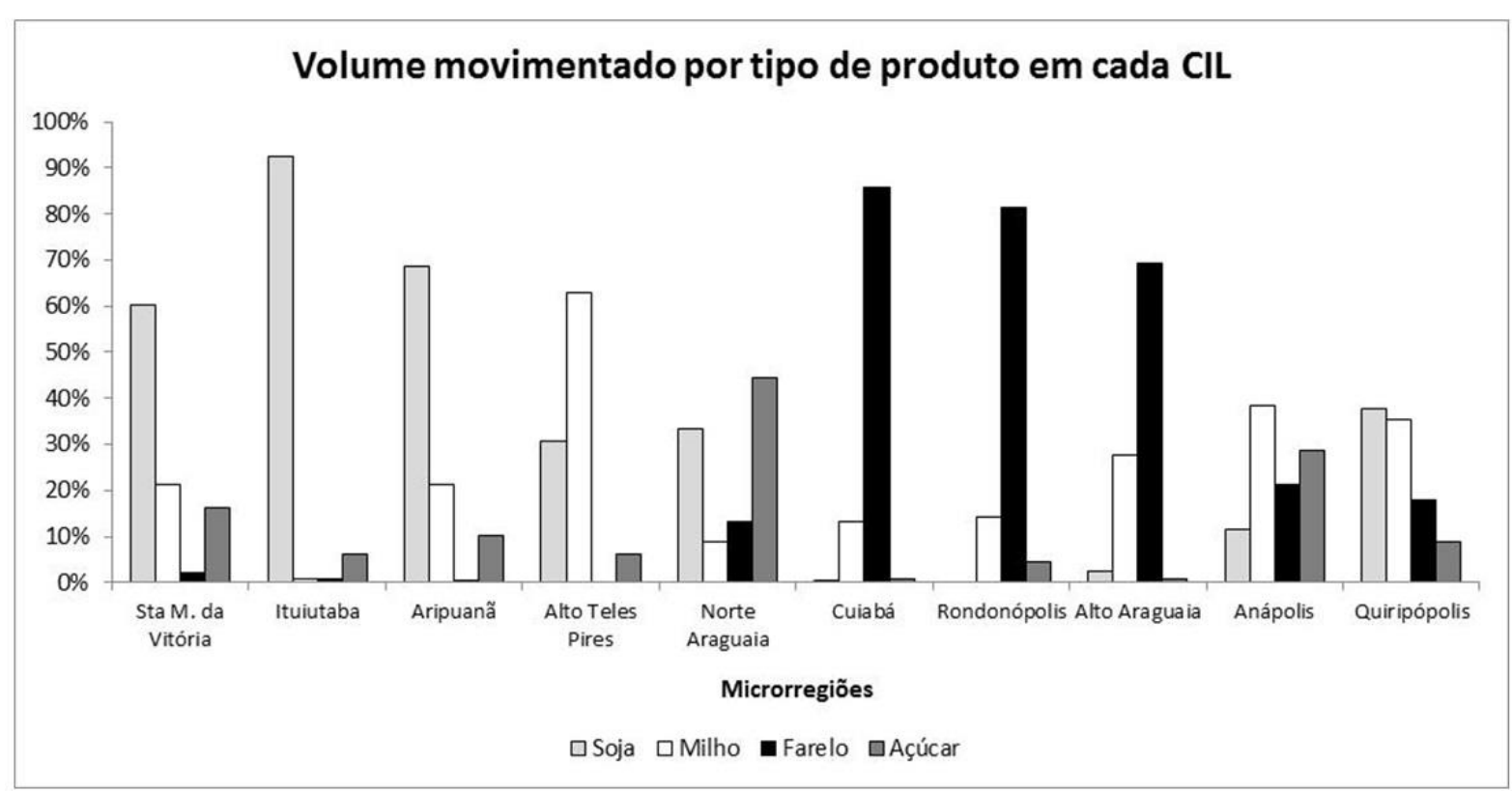

Figura 3. Volume percentual de movimentação de cada produto nos CILs abertos

O CIL da microrregião de Ituiutaba é quase exclusivamente dedicado à soja, processando apenas $6,2 \%$ de açúcar, $0,7 \%$ de milho e $0,6 \%$ de farelo. Em contrapartida, os CILs de Quirinópolis e Anápolis têm uma movimentação de carga mais equilibrada, que se divide, respectivamente, da seguinte maneira: $37,8 \%$ e $11,6 \%$ para soja; $35,3 \%$ e $38,5 \%$ para milho; $18,1 \%$ e $21,1 \%$ para o farelo de soja; e, $8,9 \%$ e $28,1 \%$ para o açúcar. Embora a soja represente $45 \%$ do volume total transportado, ela não é processada pelo CIL da microrregião de Rondonópolis e representa apenas 0,004\% do volume processado pelo CIL de Cuiabá.

O farelo de soja, por sua vez, apesar de representar mais de $80 \%$ dos volumes movimentados pelos CILs de Cuiabá e Rondonópolis, tem movimentação reduzida nos CILs de Santa Maria da Vitória (2\%), Ituiutaba $(0,6 \%)$ e Aripuanã $(0,1 \%)$, não havendo movimentação deste produto em Alto Teles Pires. O milho tem movimentação em todos os CILs, definindo o menor percentual processado em Ituiutaba $(0,7 \%)$ e o maior em Alto Teles Pires (63\%). O açúcar também é movimentado por todos os CILs, representando $0,7 \%$ da movimentação em Alto Araguaia e 0,8\% em Cuiabá e, no outro extremo, 44\% em Norte Araguaia.

Destaca-se que, embora determinado produto possa vir a ter baixa participação percentual em um dado CIL, é importante analisar também seu volume de toneladas processadas, pois este pode ser eventualmente expressivo para o produto. Por exemplo, a movimentação de açúcar em Aripuanã representa apenas 10,3\% (equivalente a aproximadamente 2 milhões de t) da movimentação total do CIL. Entretanto, o volume de açúcar processado pela estrutura é superior ao total movimentado pelos CILs de Ituiutaba (1 milhão de t), Alto Teles (mais de 1,5 milhões de t), Norte Araguaia (1 milhão de t) e Anápolis (1 milhão de t).

Além disso, é importante estimar os custos decorrentes da utilização dessas estruturas. Verificou-se que o custo total de transporte a elas associado é de 7,4 bilhões de reais. Deste total, $37 \%$ estão relacionados ao transporte da soja (que representa $45 \%$ do volume total), $26 \%$ ao milho, $23 \%$ ao farelo de soja e $14 \%$ ao açúcar.

Nota-se que a distribuição percentual dos custos converge com a participação de cada produto na movimentação total, sendo que os produtos com maior movimentação representam a maior parcela de desembolso. Entretanto, o maior custo por tonelada movimentada é o do açúcar ( $\mathrm{R} \$ 252,02)$, seguido pelo milho ( $\mathrm{R} \$ 202,57)$, farelo de soja $(\mathrm{R} \$ 182,92)$ e soja ( $\mathrm{R} \$ 143,81)$. Destaca-se que o custo unitário médio de movimentação é de $\mathrm{R} \$ 176,60 / \mathrm{t}$, sendo a movimentação de soja, portanto, mais eficiente.

Os custos totais (considerando os quatro produtos selecionados) associados à movimentação por CIL em cada uma das microrregiões consideradas variam de R\$ 126 milhões em Ituiutaba a R \$ 2,9 bilhões em Aripuanã (valor 23,1 vezes maior). Destaca-se que os três CILs com maior movimentação ( $68 \%$ do total acumulado), foram também aqueles que mais contribuíram para o custo total, sendo: $39,3 \%$ para Aripuanã, 12,5\% para Quirinópolis e 11,1\% para Rondonópolis.

Contudo, como este estudo está voltado para uma análise estratégica, é importante avaliar também a economia total proporcionada por cada CIL em relação ao transporte direto, feito pelo modo rodoviário; bem como relacioná-la aos custos do transporte efetuado por meio destas estruturas. $\mathrm{O}$ custo total de transporte considerando fluxos diretos e aqueles por meio dos CILs foi de R \$ 25,187 bilhões, sendo que $29,6 \%$ deste montante se refere ao transporte por meio de CILs e o restante está relacionado ao transporte direto. Destaca-se que a economia obtida pelo uso destas estruturas é de $\mathrm{R} \$ 1,61$ bilhão, sendo esta equivalente a $22 \%$ dos custos de transporte por meio dos CILs e $6 \%$ do total.

O CIL que acumula o maior volume de economia global é o de Aripuanã - MT (84,3\% do total), seguido por Santa Maria da Vitória - BA (3,9\%) e Norte Araguaia - MT (3,2\%). A economia dos demais CILs varia de $0,4 \%$ em Alto Araguaia - MT a 2,2\% em Anápolis - GO. A relação existente entre os custos e as economias correspondentes a cada CIL é apresentada na Figura 4.

Ressalta-se que as economias são obtidas ao trocarmos o transporte direto pelo transporte via CIL. Embora os percentuais de economia pareçam pequenos, destaca-se que o montante a eles relacionado está na ordem dos milhões. 


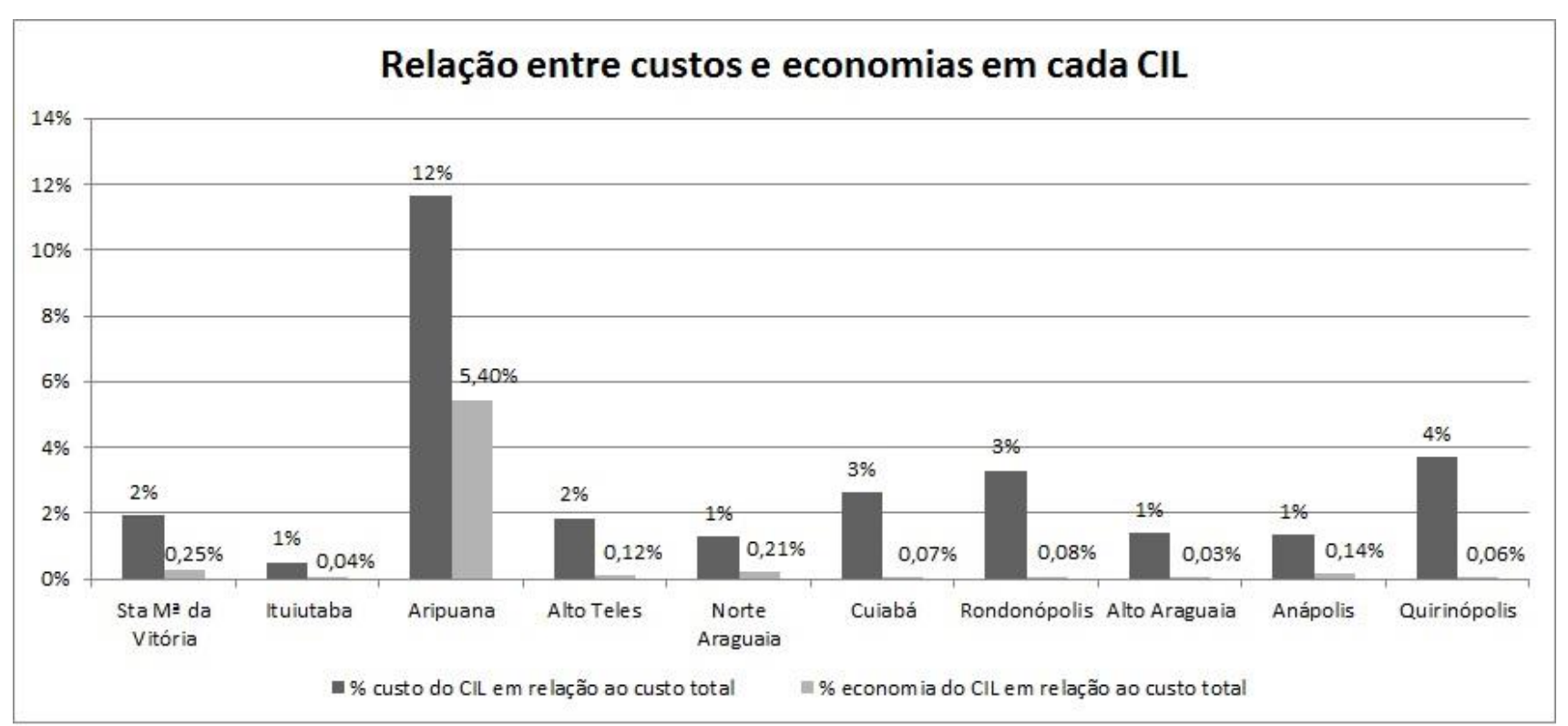

Figura 4. Relação entre o custo e a economia associados à utilização dos CILs

Assim, para o caso estudado, a economia proporcionada pelo CIL da microrregião de Aripuanã (cerca de 1,36 bilhões) seria suficiente para viabilizar, financeiramente, a utilização do CIL de Quirinópolis (o de segundo maior custo) e de qualquer outro CIL com custo inferior a R $\$ 426$ milhões.

Percebe-se, então, que o modelo de Guimarães (2015) permite indicar as regiões de abertura dos CILs (localização), a quantidade de cada tipo de produto a processar (alocação), seus custos de transporte e a economia advinda da utilização deste tipo de estrutura. Isto é fundamental para o planejamento estratégico de transportes, pois permite determinar com certo grau de precisão a capacidade adequada de um CIL para atender a cada tipo de produto a ele associado (caso os dados utilizados sejam confiáveis e desconsiderando-se as variações mercadológicas sobre as quais os gestores não têm poder de influência). Permite, ainda, que se faça uma definição mais precisa dos atributos de um CIL, a saber, quantidade e tipos de equipamentos necessários, $l a-$ youts, tipos de estruturas de armazenagem e respectivas capacidades, dentre outros.

Além disso, o modelo auxilia também na determinação dos critérios necessários para o desenvolvimento do plano de negócios da estrutura e nos estudos de viabilidade técnica, financeira, econômica e ambientais - EVTEAs. Ressalta-se, entretanto, que os resultados obtidos por meio da aplicação do modelo variam de acordo com a base de dados utilizada.

Dessa forma, os resultados obtidos neste trabalho podem ser considerados os melhores possíveis, diante da base de dados selecionada para a aplicação. Entretanto, um refinamento da mesma pode levar a resultados ainda mais precisos e eventualmente distintos dos atuais, indicando, por exemplo, localidades diferentes para a instalação de CILs.

Finalmente, é importante ressaltar que o reduzido tempo de CPU gasto pelo CPLEX para resolver o cenário em teste, ou seja, 0,4 segundos, não é um indicativo da dificuldade prática de resolução do problema. Com o aumento do número de produtos, aumentam também, fortemente, a demanda por memória RAM e o tempo de resolução. Se memória adicional e um processador mais rápido não esti- verem disponíveis, a opção seria, então, desenvolver e implementar um algoritmo de solução específico para o problema, deixando de lado o uso do CPLEX como um sistema autônomo.

Neste caso, seriam desenvolvidos algoritmos exatos, possivelmente de decomposição (ver Vanderbeck e Wolsey, 2010), que explorariam a fundo a estrutura matemática do problema com o intuito de avançar (para além do que o CPLEX atualmente consegue), o estado-da-arte na resolução do mesmo. Este algoritmo envolveria ainda, como é mandatório nesses casos, o uso de heurísticas especificamente desenvolvidas para o problema tratado. Alternativamente, se a dimensão das instâncias consideradas se mostrar proibitiva para os algoritmos exatos, metaheurísticas passariam a ser a opção de escolha para resolver o problema.

\section{CONSIDERAÇÕES FINAIS}

Este trabalho descreve uma aplicação do modelo de localização-alocação proposto por Guimarães (2015). Especificamente, um caso teste foi resolvido envolvendo quatro produtos importantes para o agronegócio brasileiro e a utilização da base de dados georreferenciada do PNLT. Os resultados obtidos sugerem a abertura de 10 CILs e identificam as microrregiões em que estes devem ser estabelecidos. Além disso, definem os produtos e os volumes de carga associados a cada CIL, minimizando assim o custo total de transporte. Ressalta-se, contudo, que as microrregiões indicadas para abertura de CILs são aquelas que minimizam os custos totais, em função dos valores de custos e capacidades disponibilizadas para o estudo. Portanto, uma alteração nesses dados pode levar a resultados distintos.

Como as principais limitações deste trabalho, apontam-se: o uso de dados secundários e estimados; o fato de terem sido considerados apenas quatro produtos da matriz de cargas brasileira; não terem sido incorporadas na base de dados georreferenciada as obras sugeridas por programas e planos governamentais recentes, como o PIL; não serem considerados os custos de eventuais transbordos intermodais após a movimentação nos CILs; e não serem considerados os custos de instalação de CILs. É importante destacar que os aspectos fiscais e tributários não foram considerados 
neste trabalho, o que pode ser considerado uma limitação visto que influenciariam diretamente nos custos de transporte e poderiam alterar a indicação das localizações para abertura dos CILs. Assim, sugere-se que este aspecto seja incorporado em estudos futuros.

Além disso, recomenda-se uma investigação considerando: grupos adicionais de produtos do PNLT; restrição de recursos para investimento; custos diferenciados por tipo de produto; custos de transbordo pós CIL; novos investimentos em infraestrutura (previstos nos planos e programas recentes); as opções de cabotagem e de dutos (para os produtos que as permitam, tais como combustíveis e derivados); imposição de uma capacidade máxima para a utilização de um determinado CIL; e/ou a inclusão do parâmetro tempo, ou seja, a identificação do momento em que um CIL deverá ser aberto. Ademais, poderia ser feita uma análise de sensibilidade nos parâmetros, estabelecendo-se diferentes cenários para avaliação. Salienta-se que se pode fazer necessária a atualização de dados e custos adicionais e, possivelmente, ajustes nas matrizes de O/D.

Destaca-se que, apesar de o modelo atender as demandas par-a-par, ele ainda carece de restrições que garantam aos agentes envolvidos utilizar sempre, dentre os CILs abertos, aqueles que lhes sejam mais favoráveis (ou seja, que ofereçam a maior vantagem econômica para transportar seus produtos). Isto significaria escolher aquele CIL que lhes proporciona o menor custo de transporte e não aquele que leva ao menor custo de transporte global, como imposto pelo modelo. Assim sendo, o desenvolvimento e a inclusão dessas restrições no modelo devem ser objeto de trabalhos futuros.

Uma nota final é que, vencidos os desafios descritos acima, elementos de Programação Estocástica deveriam ser incorporados ao modelo, visto que se trabalhou aqui com projeções de demandas futuras.

\section{AGRADECIMENTOS}

Os autores agradecem ao Conselho Nacional de Desenvolvimento Científico e Tecnológico (CNPq) e a Coordenação de Aperfeiçoamento de Pessoal de Nível Superior (CAPES) pelo suporte financeiro para desenvolvimento deste trabalho.

\section{REFERÊNCIAS}

ANTT - Agência Nacional de Transportes Terrestres (2016) Multimodal. Disponível em:

<http://www.antt.gov.br/index.php/content/view/4963/Multimod al.html>. Acesso em: abr/2016.

Almur, S. A., Kara, B. Y. e Karasan, O. E. (2012) Multimodal hub location and hub network design, Omega, v. 40, p. 927 939. doi:10.1016/j.omega.2012.02.005

Almur, S. e Kara, B.Y. (2008) Network hub location problems: The state of the art. European Journal of Operational Research, v. 190 , p. 1 -21. DOI: 10.1016/j.ejor.2007.06.008

ANTAQ (2013) Plano Nacional de Integração Hidroviária PNIH: Relatório de Metodologia. Desenvolvimento de Estudos e Análises das Hidrovias Brasileiras e suas Instalações Portuárias com Implantação de Base de Dados Georreferenciada e Sistema de Informações Geográficas. Brasília/DF.
Arnold, P., Peeters, D. e Thomas, I. (2004) Modelling a rail/road intermodal transportation system. Transportation Research Part $E$, v. 40 , n. 3, p. 255 - 270. DOI:10.1016/j.tre.2003.08.005

Bhattacharya, A., Kumar, S. A., Tiwari, M. K. e Talluri, S. (2014), An intermodal freight transport system for optimal supply chain logistics, Transportation Research Part $C$, v. 38, p. 73 - 84. DOI: $10.1016 /$ j.trc.2013.10.012

BNDES - Banco Nacional de Desenvolvimento (2011), Corredor Bioceânico Ferroviário - Estudos Técnicos Referentes ao Eixo de Capricórnio. In: Pesquisas e Estudos Técnicos Destinados à Avaliação Técnica, Econômico-Financeira e JurídicoRegulatória de Soluções Destinadas a Viabilizar o Sistema Logístico Ferroviário de Carga entre os Portos no Sul/Sudeste do Brasil e os Portos do Chile, Volume 10 - Tarifas. Brasília/DF.

Brasil (1998) Lei no 9.611, de 19 de fevereiro de 1998. Dispõe sobre o Transporte Multimodal de Cargas e dá outras providências. Brasília/DF.

Brasil (2000) Decreto ${ }^{\circ} 3.411$, de 12 de abril de 2000. Regulamenta a Lei $n^{\circ}$ 9.611, de 19 de fevereiro de 1998, que dispõe sobre o Transporte Multimodal de Cargas, altera os Decretos nos 91.030, de 5 de março de 1985, e 1.910, de 21 de maio de 1996, e dá outras providências. Brasília/DF.

Campbell, J. F. e O'Kelly, M. E. (2012) Twenty-Five Years of Hub Location Research. Transportation Science, v. 46, n. 2, p. 153 - 169. DOI: $10.1287 /$ trsc. 1120.0410

Chopra, S. e Meindl, P. (2011) Gestão da Cadeia de Suprimentos - estratégia, planejamento e operação. 4a edição, São Paulo: Pearson-Prentice Hall.

CNT e COPPEAD (2002) Transporte de Cargas no Brasil: Ameaças e Oportunidades para o Desenvolvimento do País Diagnóstico e Plano de Ação. In: Relatório da Confederação Nacional do Transporte - CNT, Centro de Estudos em Logística do Instituto de Pós-Graduação e Pesquisa em Administração COPPEAD e Universidade Federal do Rio de Janeiro - UFRJ, Rio de Janeiro/RJ.

CNT - Confederação Nacional dos Transportes (2014) Plano CNT de Transporte e Logística - 2014. Disponível em: http://www.cnt.org.br/Paginas/Plano-CNT-deLog\%C3\%ADstica.aspx. Acesso: jan/2015.

Correa, V. H. C. e Ramos, P. (2010) A precariedade do transporte rodoviário brasileiro para o escoamento da produção de soja do Centro-Oeste: situação e perspectivas. Revista de Economia e Sociologia Rural, v. 48, n. 2, p. 447 - 442. http://dx.doi.org/10.1590/S0103-20032010000200009

Costa, M. B. B. (2014) Utilização De Modelo De LocalizaçãoAlocação Para Identificação De Zoneamento Logístico Integrado Ao Planejamento Estratégico De Transportes. Tese Doutorado em Engenharia de Transportes, Universidade Federal do Rio de Janeiro. Rio de Janeiro, Brasil.

Crainic, T. G. e Laporte, G. (1997) Planning models for freight transportation. European Journal of Operational Research, v. 97, p. 409 - 438. DOI:10.1016/S0377-2217(96)00298-6

Daskin, S. M., Snyder, L. V. e Berger, R. T. (2003) Facility Location in Supply Chain Design. Department of Industrial Engineering and Management Sciences Northwestern University, Evanston, Illinois, U.S.A. Working Paper No. 03-010. 
Diniz, C. C.(2006) Dinâmica espacial e ordenamento do território. Comissão econômica para a América Latina e o Caribe - CEPAL. Acesso: 17/09/2014.

Dubke, A.F. (2006) Modelo de Localização de Terminais Especializados: um Estudo de Caso em Corredores de Exportação da Soja. Tese de Doutorado em Engenharia de Produção, Pontifícia Universidade Católica - PUC: Rio de Janeiro/RJ.

EPL - Empresa de Planejamento e Logística (2014), Programa de investimentos em logística. Brasília. Disponível em: <http://www.epl.gov.br/index.php>. Acesso em: mar/2014.

Farahani, R. Z., Hekmatfar, M., Arabani, A. B. e Nikbakhsh, E. (2013) Hub location problems: A review of models, classification, solution techniques, and applications. Computers \& Industrial Engineering, v. 64, p. 1096 - 1109. DOI: 10.1016/j.cie.2013.01.012

Fleury, P. F. (2011) Infraestrutura - sonho ou realidade? In: $8^{\circ}$ Fórum de economia da Fundação Getúlio Vargas, São Paulo.

Fleury, P. F. (2003) Terceirização logística no Brasil. In: FIGUEIREDO, K. F.; FLEURY, P. F.; WANKE, P. (Eds.) Logística e gerenciamento da cadeia de suprimentos: planejamento do fluxo de produtos e dos recursos. São Paulo: Editora Atlas.

Geoffrion, A.M. e Graves, G.W. (1974) Multicommodity Distribution System Design by Benders Decomposition. Management Science, n. 20, p. 822 - 844. DOI:

$10.1287 / \mathrm{mnsc} .20 .5 .822$

Groothedde, B., Ruijgrok, C. e Tavasszy, L. (2005) Towards collaborative, intermodal hub networks: A case study in the fast moving consumer goods market. Transportation Research Part $E$, v. 41 , n. 6, p. 567 - 583. DOI:10.1016/j.tre.2005.06.005

Guimarães, V. de A. (2015) Modelagem matemática para localização-alocação de centros de integração logística considerando as demandas par-a-par. Dissertação de mestrado em Engenharia de Transportes. PET/COPPE/UFRJ: Rio de Janeiro.

Ishfaq, R. e Sox, R. C. (2011) Hub location-allocation in intermodal logistic networks. European Journal of Operational Research, v. 210, p. 213 - 230. DOI:10.1016/j.ejor.2010.09.017

Limbourg, S. e Jourquin, B. (2009) Optimal rail-road container terminal locations on the European network. Transportation Research Part E, v. 45, n. 4, p. $551-563$.

DOI:10.1016/j.tre.2008.12.003

Lopes, S. S., Cardoso, M. P. e Piccinini, M. S. (2008) O Transporte rodoviário de carga e o papel do BNDES. Revista do BNDES, v. 14 , n. 29 , p. $35-60$.

Meisel, F., Krischstein, T. e Bierwirth, C. (2013) Integrated production and intermodal transportation planning in large scale production-distribution-networks. Transportation Research Part $E$, v. 60 , p. $62-78$. http://dx.doi.org/10.1016/j.tre.2013.10.003

Melo, M. T, Nickel, S. e Saldanha-da-Gama, F. (2009) Facility location and supply chain management: a review. European Journal of Operational Research, v. 196, p. 401 - 412. DOI:10.1016/j.ejor.2008.05.007

Ministério da Agricultura - MA (2015) Agronegócios.
Ministério dos Transportes - MT (2007) Plano Nacional de Logística e Transportes: Sumário executivo. Brasília.

Ministério dos Transportes - MT (2009) Relatório Executivo PNLT. Brasília.

Ministério dos Transportes - MT (2012) Projeto de reavaliação de estimativas e metas do PNLT: Relatório Final. Brasília.

Moori, R. G. e Riquetti, A. (2014). Estação de Transbordo de Cargas como Mediador da Logística de Fertilizantes. Revista de Administração Contemporânea, v. 18, n. 6, p. 748 - 771. DOI: 10.1590/1982-7849rac20141189

Padula, R. (2008) Transportes - Fundamentos e propostas para o Brasil. Brasília: Confea. Disponível em: <http://www.confea.org.br/media/Livro_Transportes.pdf $>$.

Quadros, S. G. R. e Nassi, C. D. (2015) An evaluation on the criteria to prioritize transportation infrastructure investments in Brazil. Transport Policy, v. 40, p. 8 - 16. DOI:10.1016/j.tranpol.2015.02.002

Racunica, I. e Wynter, L. (2005) Optimal location of intermodal freight hubs. Transportation Research Part B, v. 39, n. 5, p. 453 - 477. DOI:10.1016/j.trb.2004.07.001

Shapiro, A., Dentcheva, D. e Ruszczyński, A. (2009) Lectures on stochastic programming: modeling and theory. MPS-SIAM series on optimization. Society for Industrial and Applied Mathematics: Philadelphia.

Silva, E. L. e Menezes, E. M. (2001) Metodologia da pesquisa e elaboração de dissertação. $3^{\mathrm{a}}$ ed. rev. Atual. Florianópolis: UFSC.

Sirikijpanichkul, A. e Ferreira, L. (2005) Multi-Objective Evaluation of Intermodal Freight Terminal Location Decisions. In: Proceedings of the 27th Conference of Australian Institute of Transport Research (CAITR), Queensland University of Technology.

Slack, N., Chambers, S. e Johnnston, R. (2009) Administração da Produção. $3^{\mathrm{a}}$ ed. São Paulo: Editora Altas.

USP - Universidade de São Paulo (2012) Sistema de Informações de Fretes - SIFRECA. Grupo de Pesquisa e Extensão em Logística Agroindustrial - ESALQ-LOG, Piracicaba/SP.

Vanderbeck, F. e Wolsey, L. A. (2010) Reformulation and Decomposition of Integer Programs. In: 50 Years of Integer Programming 1958-2008 - From the Early Years to the State-ofthe-Art. Orgs: Junger, J et al.. Springer.

Vasconcelos, A. D., Nassi, C. D. e Lopes, L. A. S. (2011) The uncapacitated hub location problem in networks under decentralized management. Computers and Operational Research, v. 32, p. 467 - 984. DOI: 10.1016/j.cor.2011.03.004

Vidovic, M., Zecevic, S., Kilibarda, M., Vlajic, J., Bjelic, N. e Tadic, S. (2011) The p-hub model with hub-catchment areas, existing hubs, and simulation: A case study of Serbian intermodal terminals. Networks and Spatial Economics, v. 11, n. 2, p. 295 - 314. DOI:10.1007/s11067-009-9126-7 